\title{
Research on Multi Agent Cooperative Mechanism of University Emergency Management from the Perspective of Big Data
}

\author{
Li Wang \\ School of Mechanical and Electronic Engineering, Wuhan University of Technology, Wuhan 430070, \\ China
}

15152105@qq.com

\begin{abstract}
The frequent occurrence of emergencies in universities not only threatens the stability and development of the campus, but also challenges the traditional emergency management system and the model. Combined with the characteristics of the era of big data, this paper comprehensively uses emergency life cycle theory, synergetic theory, multi agent theory, and puts forward multi agent cooperative mechanism of emergency management, two big data case libraries, four cycles, three disposal agents, based on the classification of emergencies universities. And then the internal operation mode of the mechanism is interpreted, which provides a new method for the university emergency response theory.
\end{abstract}

Keywords: Big data; Emergency in universities; Cooperative processing mechanism; Multi agent theory.

\section{Introduction}

Under the new media environment, the emergency response of universities is becoming more and more complex [1]. The emergency in universities is that due to colleges and universities a variety of internal and external factors, to the staff of colleges and universities as the main body, suddenly occur within a relatively short time, and quickly evolved for larger scale, causing casualties or major property damage, and even endanger the image and reputation of the event [2]. By definition, emergency in universities has the characteristics of "time burst, spatial wide area, cause complexity, type diversity, and high risk" and so on. And the traditional single disposal of the main, top-down disposal mechanism, temporary disposal program cannot solve the disposal of university emergencies. Based on literature review. Henstra (2010) specify 30 factors of effective local emergency management procedure, and integrate these key factors in a framework, thus provide a method for the evaluation and implementation of emergency management procedure [3]. Jackson and Sullivan (2011) describe the emergency response system's reliability analysis process, propose a method of assessing emergency response system, and validate it with a case. Considering the factors affecting the capability of colleges' emergency management [4]. Ji Hao, Su Bing and Lv Mei (2012) construct colleges' emergency management system based on emergency management organizational mechanism risks, emergency prevention capability risks, emergency handling capability risks, and emergency recovery capability risks [5]. Liu Qinghua et. al. (2012) construct colleges' emergency management system according to Emergency handling process with SMART principle [6]. Biological chemist Tony Cass (1988) published a paper used to deal with the "big data" software package HiQ in the journal Science [7]. Since then, the application of big data is more and more common, and puts forward new ideas for public management. The "4V" characteristics of big data promote great changes for the traditional emergency response mode. Big data technology can be used to deal with the complex multi-source heterogeneous data into the effective information of decision value, making it possible for the analysis and decision making under high uncertainty and high time pressure. Therefore, in the era of big data, universities must be integrated to improve the existing emergency management mechanism, the formation of an open, organic and reasonable, coordinated operation of the response mechanism, so as to improve the risk prevention capabilities of universities. 


\section{Analysis on the Problems of the Traditional University Emergency Handling Mechanism}

\subsection{The emergency response plan was passive, lacking the internal driving force}

Universities generally developed a campus normal emergency plan to protect students' lives and property safety. But once in the face of unexpected events have not occurred, the inherent emergency plan will be in a passive response phase. And the leadership of the school is the power source of the emergency disposal mechanism in traditional, operation mode is also top-down command response. Thus, college students management mechanism of public emergencies often change over the leadership of the school will. From the viewpoint of information, even the students, counselors, faculties, school security departments, as well as the relevant leaders get a key information, they can't alarm in time, and are unable to promptly formulate corresponding countermeasures. Reported to the relevant departments, they are often easy to delay the disposal of the best time. Once the sudden mass incidents occurred, to convene school leaders and related personnel is not only a waste of time, but also an obstacle to maximize the ability of mutual cooperation under the condition of handling emergencies. The internal drive mechanism has not yet formed and the contingency ability is insufficient, which is not conducive to the disposal of unexpected public events in universities.

\subsection{Lack of permanent multi collaborative emergency response organizations, resulting in the phenomenon of "emergency information island"}

University emergency management organization system is unsound, this mainly displays in the following two aspects: firstly, taking the university as the only subject, the multi subject of university emergency management has not yet formed a joint force. Secondly, the organization of the emergency handling of emergencies is mostly temporary and loose composition. In general, when the unexpected events occurred, emergency management team will be composed by the school leaders temporarily. With the end of the event, the team will be dissolved at the same time. This temporary and loose organization is congenitally deficient in facing the emergency management of unexpected events. It can be only used for a short-term effect.

\subsection{Lack of normalization of early warning and disposal of information systems, control of the disposal efficiency}

Frequent emergencies in universities and its great harm make us to realize the necessity and urgency of establishing emergency response system. But at present, the emergency treatment system in universities is backward. Although schools have already put into a certain human and material resources in the emergency rescue facilities, communications facilities, emergency personnel quality promotion and so on, compared to the western developed countries, the overall situation of the investment is still not optimistic, which still requires the attention of leaders at all levels and relevant departments. Constructing effective and reasonable system is the necessary precondition of emergency management in universities. While a considerable part of the university leaders cannot fully understand the whole content and core of emergency management, thus they usually ignore the importance of prevention and early warning mechanisms.

\section{Construction of emergency handling mechanism in universities}

\subsection{Two big data case libraries}

(1) Emergency case database

In order to change the passive situation into the active situation, the university should build the emergency case library in advance. However, unexpected events have their contingency factors, the university cannot do nothing but wait for the event happens. Then we can use the big data, and collect the case between universities timely.

(2) Emergency case database

The case library is prepared for the timely conversion to the preparation of the plan. It is an action plan developed for the unexpected events in advance. It ensures that the emergency process is 
evidence-based. It also plays a guiding role in response to the emergency organization and effectively prevents the process of chaos. It is the basic guarantee for the timely and effective response action of emergency response. As shown in Figure 1, the plan includes five elements.

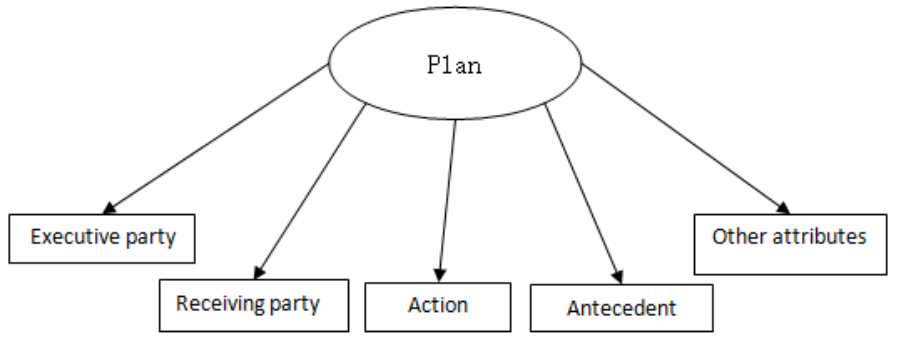

Figure 1 The Transformation Elements Of The Case Library

\subsection{Four cycles}

(1) Incubation period. Incubation period is longer than other stages. The outbreak of public emergencies are the same as the development of everything in the world and it needs to undergo a complete process of quality change to qualitative change. And in this process, the process of the outbreak is the accumulation of the energy needed to process. Once the energy accumulates to a certain level, as long as there is a "spark", it will explode and bring harm to the society.

(2) Outbreak period. When the energy is accumulated to a certain degree, it must be expressed in a certain way. Energy cathartic process is such an outbreak of public emergencies qualitative change. The main feature of this period is the duration is short, but the intensity is great.

(3) Spreading period. The spreading period means after the outbreak of the emergency, with the continuing impact of the disaster, the destructive forces are still continuing to function. In the strict sense, there is no specific boundary differentiation between the outbreak and the influence period, and there are many links between them. During the period, the social impact caused by the unexpected events will affect all levels of the society and the part. Conversely, their different responses will produce huge damage to the community. If we cannot eliminate its negative effects, and prevent greater losses caused, it will cause greater harm to society and lead to more public emergencies. If that's the case, things will be out of control and have long-term effects. Therefore, we need to work together, and use our knowledge to take effective measures to minimize its negative impact.

(4) Ending period. In a period of time after the outbreak of public emergencies, with human or natural control of the crisis, this public emergency has moved to its final stage - the ending period. Different angles have different views about the specific time of the ending period. From the perspective of management science, the main symbol of the end is that the community is recovering from the damage. In the perspective of the process of the development of the emergency, the real end of the event is the harm and impact caused by the sudden incident are eliminates completely.

\subsection{Three disposal agents}

The reasonable function agent organization structure of the multi agents cooperative decision system of the emergency disposal has directly influence on the effect and success of the emergency disposal. The function subjects of the multi agents cooperative decision making system in the emergency disposal of the university are divided into the following three parts

(1) Decision making command agent. In the event disposal, the decision - making command is responsible for the function of the decision - making and the allocation of the building function. It requires the ability to integrate resources. The decision-making command agent of the emergency events can make judgments and responses quickly and efficiently though identifying the status or type of the of emergency event. Decision making command agent is based on the multi agents cooperative strategy of the multi agents cooperative intelligent decision making system. It can give orders the corresponding event information to each of the functions of participating in the emergency response. At the same time, based on the site feedback information of the various functional agents, it 
can make an accurate decision after processing, and analyzing on the information. This system can ensure that each function agent accurately perform its work and finally reach the target.

(2) Emergency disposal group. Emergency disposal group is based on the function, the division of labor in the process of the disposal process according to the different tasks, or it is the agents combination of the disposal of the specific task. Emergency disposal group is responsible for sending the feedback information from the disposal site to the decision making command. Emergency disposal group is the facial features and hands and feet of a system. The main body of each function in the emergency disposal group has their own professional direction, and the internal resources and operation mechanism of each function agent is the basis of the function of the group. In the whole process of the event disposal, information is communicated between the functional agents, the functional agents and the intelligent cooperative agents, decision making command agents. They coordinate and share the resources to ensure the success of the disposal of the target.

(3) Intelligent cooperative agent. Intelligent cooperative agent bears the mission to provide expert advice, information collection and judged, determination of functional agent intervention strategy, the establishment and improvement of the cooperative mechanism to the decision making command agent. Intelligent cooperative agent is the brain of the system. The main body of the intelligent cooperative agent has the knowledge of various fields as well as the advanced technical means. It can provide reliable experience and efficient information support for the scientific decision making of each function in the event management. It provides expert advice when it comes to the strategy functional agent intervention and the establishment and improvement of the cooperative mechanism and a strong solution support for event handling and execution process. First of all, when the original information arrives, it researches on the implementation of event category and definite various functions of the disposal of the property in the event. When handing the event, it supervises the function of each agent. At the same time, it provides knowledge, information and other decisions and actions that required for expert advisory services. Finally, the intelligent collaboration agent is also responsible for the knowledge and experience of the event management process, which includes the coordination mechanism of the upgrade, the function of the nuclear intervention strategy and so on. Intelligent collaboration agent is the soul of multi agents collaborative intelligent decision making system. The integration of knowledge structure and advanced technology in a number of specialized fields is a powerful functional agent subject. Although its main body is not involved in the event of on-site disposal, the effective play of its function will directly influence the decision making of the decision making command agent and the emergency disposal group at the scene. It is the key to realize the efficient operation and intelligence characteristic of multi agents cooperative intelligent decision making system.

\subsection{The operating mechanism}

As shown in Figure 2, the disposal mechanism of emergency management in universities is composed of "two big data case libraries, four cycles, three disposal agents". 


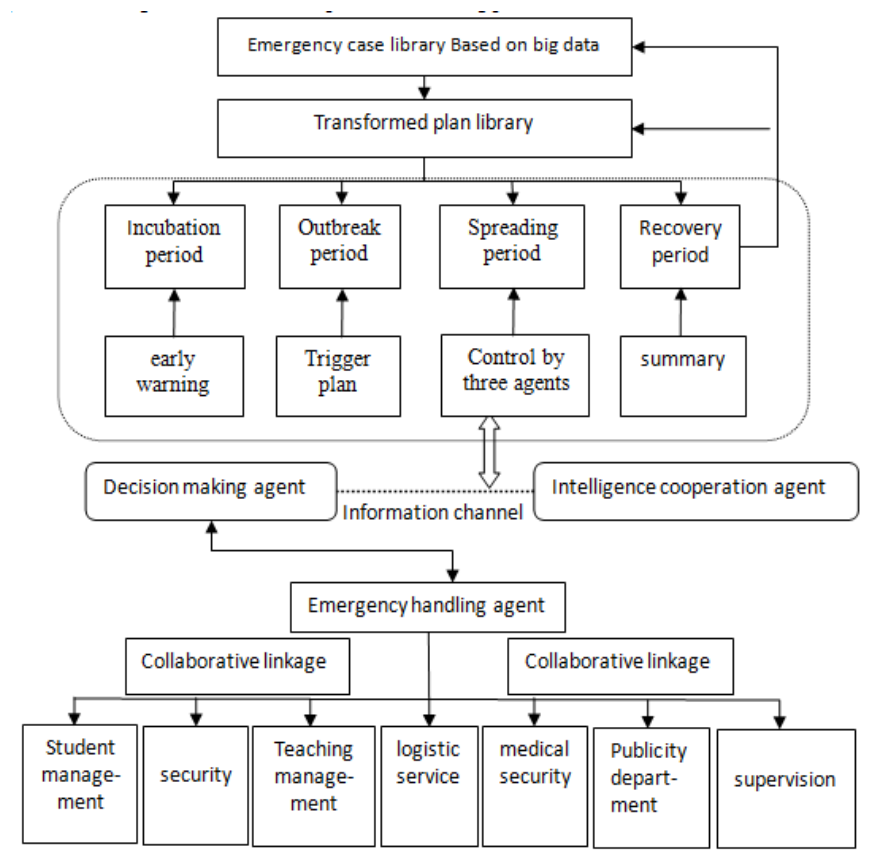

Figure 2 The "Two Four Three" Collaborative Mechanism of Emergency Management

First of all, through the construction of emergency public opinion monitoring and control institutions, universities can form the emergency case library. And in accordance with the plan of the five elements of the form, it can be conversed into a plan library. Secondly, we should establish a comprehensive early warning and disposal mechanism to avoid the event out of control. (1) We need to identify and early warning of events at incubation period. Early warning of each emergency through the case feature comparison and call their attention on preventing the events. If the event can be resolved in the incubation period, the damage on the university will be minimal. (2) We need to trigger the corresponding plan in time at the outbreak period. If we can identify the nature of the event accurately, we can trigger the corresponding plan and respond timely. (3) We need to strengthen the three agents control at the spreading period. The ideal state is to eliminate the event in the first two stages of the University. If the emergency development to the third stage, it is important to start the three agents management and control mechanism because it does the biggest damage to the University. Universities should lead to the recovery period as soon as possible, shorten the time of the spreading period. (4) We need to summarize the accident aftermath at the recovery period. First of all, we should eliminate the impact of the early events of the University. Secondly, we should sum up the reasons for the expansion of the situation and the problems in the process of emergent events and the experience of solving problems.

\section{Conclusion}

In recent years, under the traditional management mode, most of the university emergency decision-making is based on personal experience, which is very easy to produce the rigidity of the mode of emergency management and the plight of the information island. At present, the key point of emergency management in universities is to strengthen the mechanism innovation especially in the era of big data. We need to integrate all kinds of information effectively, and make full use of the existing manpower and material resources of the university so that the internal departments and external emergency departments orderly linked. We can build the "two four three" cooperative processing mechanism with the multi agents cooperative system so that we can solve the problem of information isolated island phenomenon, and make the decision of the university emergency handling process more scientific and systematic. 


\section{Acknowledgments}

This paper is supported by HubeiCollaborative Innovation Center for Early Warning and Emergency Response Technology (JD201604XYAQ4).

\section{References}

[1] Chen ping, Yu qing. A Study on Response to the Public Opinion on Unexpected Campus Incidents in the Context of New Media [J]. Jouranal of Zhejiang Sci-Tech University (Social Sciences), 2014 (03):245-248 (In Chinese).

[2] Zhang yiting, Xie Wei. Building of the Five Forces Model for University Emergency Network Public Opinion Based on Entropy Theory [J]. Journal of Intelligence, 2012 (11):19-22 (In Chinese).

[3] Henstra D. Evaluating Local Government Emergency Management Programs: What Framework Should Public Managers Adopt [J]. Public Administration Review, 2010, 70 (02): 236-246.

[4] Jackson, B. A., Sullivan Faith. Are We Prepared? Using Reliability Analysis to Evaluate Emergency Response Systems [J]. Journal of Contingencies and Crisis Management, 2011, 19 (03): 147-157.

[5] Jihao, Subing, Lvmei. Evaluation of Universities's Emeergency Management Capability Based on Risk Factors [J]. Journal of Xi'an Technological University, 2012 (07): 584-589 (In Chinese).

[6] Liu Qinghua, Li Weiwei, Wu Chong. Ability in Tackling Collegiate Emergency [J]. Journal of Yangzhou University (Higher Edycation Study Edition), 2012 (02): 82-87 (In Chinese).

[7] Tony Cass. A Handler for Big Data [J]. Science, 23 Oct 1998: Vol. 282, Issue 5389, pp. 636

[8] Huang Youfa. Information communication mechanism of emergency handling in Colleges and Universities [J]. Joutnal of Fuzhou University (Philosophy and Social Sciences), 2011 (06), 10-15 (In Chinese). 